Juan R. Cubillos-Ruiz, Jack Hoopes, Steven Fiering and Jose R. Conejo-Garcia

\title{
Inflammatory and immune responses induced by nanomaterials: challenges and opportunities for future nanotherapies
}

\begin{abstract}
The implementation of nanotechnology for therapeutic or diagnostic applications in a clinical setting will generate new mechanisms of injury and toxicological effects. To address the biological dangers of using nanomaterials in vivo, a new field of nanotoxicology will need to be developed by scientists dedicated to understanding the toxic potential of these specific materials. Common mechanisms of toxicity induced by most nanomaterials will likely involve the generation of inflammatory and immune responses, due to the spontaneous (and likely unavoidable) uptake of nanoparticles by leukocytes and subsequent cytokine release. These responses can be adequately controlled if they are properly evaluated in pre-clinical models and monitored in subsequent clinical trials. Ignoring these potential problems will limit or block the use of nanomaterials with otherwise extremely attractive physical properties. In appropriate situations, nanoparticles can also be designed to maximize their immunostimulatory capacity in diseases where immunosuppression is the rule (e.g., in cancer), or to eliminate pathological phagocytes from places of cytotoxic inflammation. Strong interactions between toxicologists, immunologists, bioengineers and clinicians directly applying nanotherapies will need to be established in order to develop predictors and interventions for unacceptable inflammatory reactions, or to use the immunostimulatory potential of nanomaterials for therapeutic purposes. A more multidisciplinary approach and stronger concern for the biological consequences and possibilities of utilizing new nanomaterials must be developed to apply the extensive array of nanoparticles generated in recent years for biomedical purposes. This approach must be developed and applied as soon as possible before funding agencies and review panels lose patience in the absence of effective clinical applications.
\end{abstract}

Nanotechnology Perceptions 5 (2009) 195-203

Nonsubscribers: purchase individual article 